\title{
Compromiso organizacional y actitud al cambio del personal que labora en la Universidad Nacional Intercultural de la Amazonia
}

\author{
Organizational commitment and attitude to the change of the personnel \\ that works in the National Intercultural University of the Amazon
}

\author{
Dulio Oseda Gago ${ }^{1,}$,",María C. Ramos Toledo², Olga A. Cirilo López ${ }^{3}$, Rocio Manrique Chávez ${ }^{4}$, Liliam Zevallos Solis ${ }^{1}$ \\ ${ }^{1}$ Universidad Nacional Intercultural de la Amazonía, ${ }^{2}$ Universidad ESAN, ${ }^{3}$ Universidad Privada del Norte, \\ ${ }^{4}$ Universidad Nacional de Huancavelica
}

\section{RESUMEN}

La presente investigación tuvo como objetivo principal correlacionar las variables compromiso organizacional y actitud al cambio en el personal que labora en la Universidad Nacional Intercultural de la Amazonia. La hipótesis: Existe correlación directa entre el compromiso organizacional y actitud al cambio en el personal que labora en la Universidad Nacional Intercultural de la Amazonia. Para tal efecto, se realizó un estudio cuantitativo, de tipo aplicativo, y nivel correlacional; se utilizó el método científico y el método hipotético deductivo, además se tomó el diseño no experimental transversal. Se empleó la técnica de la encuesta y como instrumentos los cuestionarios de encuesta para medir las dos variables de estudio. Los resultados fueron analizados en el programa estadístico SPSS v.25, cuyos resultados nos dicen que en la variable compromiso organizacional el nivel prevalente es el regular con el $68,10 \%$ y en la variable actitud al cambio el nivel prevalente fue el alto con el $78,53 \%$. También se determinó que existe una correlación directa muy fuerte $(r h o=0,946)$ altamente significativa (p-valor: $0,000<0,010$ ) entre el compromiso organizacional y actitud al cambio en el personal que labora en la Universidad Nacional Intercultural de la Amazonia. Se concluye que, a un mejor compromiso organizacional, existe también una mejor actitud al cambio en el personal que labora en la universidad y viceversa.

Palabras clave: Compromiso organizacional, actitud al cambio, superación, apertura y valentía.

\begin{abstract}
The main objective of this research was to correlate the variables organizational commitment and attitude to change in the personnel that works in the National Intercultural University of the Amazon. The hypothesis: There is a direct correlation between the organizational commitment and attitude to change in the personnel that works in the National Intercultural University of the Amazon. For this purpose, a quantitative study of the application type and correlation level was carried out; the scientific method and the hypothetical deductive method were used, and the transverse non-experimental design was taken. The survey technique was used and as instruments the survey questionnaires were used to measure the two study variables. The results were analyzed in the statistical program SPSS v.25, whose results tell us that in the organizational commitment variable the prevailing level is the regular one with $68.10 \%$ and in the variable attitude to change the prevailing level was high with the $78.53 \%$. It was also determined that there is a very strong direct correlation (rho $=0,946$ ) highly significant ( $p$-value: $0,000<0,010$ ) between the organizational commitment and attitude to change in the personnel that works in the National Intercultural University of the Amazon. It is concluded that, to a better organizational commitment, there is also a better attitude towards change in the personnel that works in the university and viceversa.
\end{abstract}

Keywords: Organizational commitment, attitude to change, overcoming, openness and courage.

Historial del artículo:

Recibido, 11 de agosto de 2017; aceptado, 4 de diciembre de 2017; disponible en línea, 05 de enero de 2018

* Profesor Principal de la Universidad Nacional Intercultural de la Amazonía, Ph.D. in Bussines Administration (USA)

Correo: dosedag@hotmail.com 


\section{INTRODUCCIÓN}

Hoy en día el talento humano presenta cada vez mayor importancia y relevancia. Si bien es cierto, las organizaciones están compuestas por múltiples personas, y estos constituyen agrupamientos sociales creados y modificados constantemente para alcanzar objetivos específicos (Blau y Scott, 1963, citado en Arias, Varela, Loli \& Quintana, 2013); surgen diversas situaciones problemáticas que deben ser atendidas desde la administración del talento humano.

Así, el eje principal de una organización es el capital humano y su eficacia se mide según la consecución de los objetivos institucionales. Es por ello que el compromiso organizacional toma relevancia ya que si las personas asumen responsabilidad para con la organización entonces la eficacia de las organizaciones aumentará logrando sus objetivos planteados.

Entonces, uno de los aspectos más relevantes e importantes en las organizaciones es el compromiso organizacional, ya que este contribuye a la mejora, logro de objetivos y compromiso del trabajador con la organización. Asimismo, consideran que el compromiso y la motivación laboral, son aspectos que suelen estar unidos y que, al ser adecuados, contribuyen positivamente a toda la organización (Ruíz, 2013).

En el Perú, la situación no es diferente; existe factores internos y externos que afectan al $78 \%$ a los trabajadores en las actividades laborales que realizan, lo que afecta su desempeño laboral y por ende su motivación para realizar sus actividades (El Comercio, 2009). Gil (2013) determinó que el $54 \%$ de los trabajadores presentaban niveles bajos de compromiso organizacional. En la misma condición se encuentra la actitud al cambio, que una forma de vida necesaria en la mayoría de las organizaciones, y en lo personal. Toda organización debe tener la capacidad para adaptarse al cambio y en consecuencia estar abierta al aprendizaje colectivo. Aunque, no hay que olvidar que la intransigencia es una reacción normal e inevitable que se presenta en el periodo de incertidumbre a lo desconocido, que se suscita de la inseguridad de lo que vendrá y que siempre dependerá de la percepción de cada individuo, por lo que se hace necesario conocer las diferentes maneras que existen para lograr superar dicha intransigencia, y de esta manera conocer la forma de lograr que las personas se adapten a aplicar los aprendizajes que resultan de un cambio.

Existen muchos aspectos a analizar que se relacionan a esta resistencia al cambio, que es necesario considerar como la confianza en sí mismos que puede obstaculizan el crecimiento y desarrollo personal, perdida de estatus, seguridad en el trabajo o ambos, los cambios administrativos y tecnológicos que amenazan con modificar las bases del poder o eliminar puestos suelen generar resistencia intensa, presión de colegas, una persona a la que no afecte directamente el cambio podría resistirse activamente a él para proteger los intereses de sus compañeros de trabajo, alteración de las tradiciones culturales, la dinámica grupal y cultural se desequilibra siempre que se transfiere, promueve o reasigna a empleados, conflictos de personalidad.

Hay que destacar, que las organizaciones al igual que los individuos, tienden a resistirse al cambio, negándose a adaptarse a las diferentes transformaciones que se suceden en su medio o entorno, interno o externo. El cambio es la variación o paso de una situación permanente a otra diferente, que consiste en moverse desde una situación actual y estable, pasando por desequilibrios e inestabilidad, a otra situación de equilibrio futuro, por esa razón mientras más grande sea el cambio, más grande es la resistencia al mismo. Teniendo en cuenta estos criterios, la investigación responde a la interrogante: ¿ Cuál es la correlación que existe entre el compromiso organizacional y actitud al cambio del personal que labora en la Universidad Nacional Intercultural de la Amazonia?

El objetivo fue determinar la correlación que existe entre el compromiso organizacional y actitud al cambio del personal que labora en la Universidad Nacional Intercultural de la Amazonia.

La hipótesis formulada fue existe correlación directa y significativa entre el compromiso organizacional y la actitud al cambio del personal que labora en la Universidad Nacional Intercultural de la Amazonia.

La investigación se justifica según la percepción de Hernández, Fernández \& Baptista, (2014) en la medida que se trata de temas reales de las organizaciones contemporáneas, muy necesarios en este contexto temporal y espacial.

Respecto a los antecedentes, se tiene a las investigaciones de Andrade, Betanzos, \& Paz (2006): Compromiso organizacional en una muestra de trabajadores mexicanos, se concluyó que hay cuatro dimensiones del compromiso organizacional: Identificación - implicación, compromiso afectivo, compromiso de continuidad (falta de alternativas) y compromiso normativo.

También se tiene la investigación de Uribe (2007) Actitud frente al cambio y su relación con el compromiso organizacional en organizaciones de la región del Maule. Este estudio concluye que existe una relación positiva entre compromiso normativo y la aceptación frente a los cambios en ambas organizaciones $(r=$ 0,$416 ; p<0,01$ ). También se reporta una correlación negativa entre compromiso afectivo y cinismo frente a 
los cambios en los empleados de la organización A ( $r$ $=-0,382 ; p<0,01)$.

La investigación realizada por García, Rojas \& Díaz (2011) relación entre el cambio organizacional y la actitud al cambio en trabajadores de una empresa de Bogotá. Se concluye que existen correlaciones significativas entre las distintas dimensiones de ambas escalas, asimismo los empleados de la organización manifestaron una actitud de favorabilidad y flexibilidad hacia el cambio.

Arenas \& Daryelis, (2013), la resistencia al cambio y el aprendizaje organizacional innovador en Instituciones Educativas. Las conclusiones clarifican que el nivel de incidencia de la resistencia al cambio se correlaciona positiva y significativamente con el aprendizaje organizacional, por lo que se plantearon estrategias gerenciales que permitan eliminar la resistencia al cambio y generar aumento del nivel de aprendizaje organizacional en las instituciones educativas.

Y el de Chávez, Cigüeñas \& Martensen (2016) la relación entre los estilos de liderazgo y las actitudes ante el cambio organizacional en una empresa de servicios. Sus conclusiones consideran que el estilo de liderazgo predominante en la empresa es el transformacional y la actitud predominante es la aceptación. Luego los estilos de liderazgo transformacional, transaccional y correctivo se relacionan de manera significativa, moderada y positiva con la actitud de aceptación ante el cambio organizacional.

Las contribuciones que se realiza, el comportamiento organizacional emerge en las formas de comunicación, se centra en conductas observables, como la comunicación entre compañeros, manejos de equipo, preparación de los diversos informes. Así mismo, se ocupa de los estados internos como la percepción, el pensamiento y la toma de decisión, entre otros. Igualmente, estudia a las personas tanto individual y grupal, en sus comportamientos al interior de una organización.

Y por otro lado tenemos, la actitud hacia el cambio, es el grado en que la gente está dispuesta a aceptar que los nuevos tiempos demandan nuevas soluciones, a contemplar alternativas diferentes, a buscar otros caminos y formas de hacer las cosas, a aprender (considerando que el aprendizaje no es la mera acumulación de información, sino la modificación de conductas), a trabajar en condiciones de incertidumbre, - estar al tanto de lo que sucede en el entorno de la organización y de la propia área de especialidad, a mejorar constantemente los productos, servicios y procesos.

Respecto a las dificultades, hubo algunos trabajadores de la universidad que se rehusaron a ser encuestados, pues creían que se trataba de una evaluación de personal y no una investigación, a pesar que se le esclareció la duda.

Las limitaciones fueron principales de orden económico, ya que la investigación fue autofinanciada en todo su sentido.

\section{MATERIAL Y MÉTODOS}

La investigación fue del tipo aplicada y de nivel correlacional.

En la investigación, se utilizó el método científico y como método específico el método descriptivo. El diseño de la investigación fue el descriptivo correlacional. Según Kerlinger y Lee (2002, p. 255) "el diseño descriptivo correlacional, es aquel diseño donde no se manipula ninguna variable, solo se miden y luego se comparan para ver qué relación existe entre éstas".

El esquema es el siguiente:

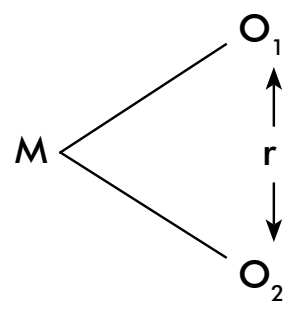

Dónde:

$M=$ Muestra

$\mathrm{O}_{1}=$ Observación de la variable 1 .

$\mathrm{O}_{2}=$ Observación de la variable 2 .

$r \quad=$ Correlación entre dichas variables.

En el caso de nuestra investigación, la población estuvo conformada por 210 sujetos, entre 114 docentes y 96 administrativos de la Universidad Nacional Intercultural de la Amazonia, Pucallpa. El Muestreo fue probabilístico, y estuvo conformado por 163 sujetos entre 88 docentes y 75 administrativos de la Universidad Nacional Intercultural de la Amazonia de Pucallpa, en el periodo lectivo 2017.

Respecto a las técnicas de recolección de datos se tuvo a la encuesta, con su instrumento el cuestionario de encuesta, el cual se aplicó a las dos variables de estudio. La confiabilidad se hizo con el alfa de Cronbach $(0,947)$ y la validez por el juicio de experto (95\%). 


\section{RESULTADOS}

En las tablas siguientes se muestra el consolidado de las variables "Compromiso organizacional" y la "Actitud al cambio", el cual se aplicó a la muestra de 88 docentes y 75 administrativos de la Universidad Nacional Intercultural de la Amazonia, Pucallpa 2017.

Según la tabla 01 se puede apreciar que los niveles de la variable $N^{\circ} 1$ Compromiso organizacional de los 88 docentes encuestados, 66 docentes que representa el $75 \%$ perciben que el compromiso organizacional es regular, asimismo 45 administrativos que es el 60\% menciona lo mismo; luego 21 docentes que es el $23,86 \%$ y 28 administrativos que es el $37,33 \%$ mencionan que el compromiso es alto; finalmente solo un docente que es $1,14 \%$ y 2 administrativos que es el $2,67 \%$ mencionaron que el compromiso es bajo. A manera de síntesis el compromiso organizacional imperante en la universidad es el regular o medio.

Según la tabla 02 se puede apreciar que los niveles de la variable $\mathrm{N}^{\circ} 2$ Actitud al cambio, de los 88 docentes encuestados, 70 docentes que representa el 79,55\% perciben que la actitud al cabio es alta, asimismo
58 administrativos que es el 77,33\% mencionan lo mismo; luego 16 docentes que es el $18,18 \%$ y 12 administrativos que es el $16 \%$ mencionan que la actitud al cambio es regular; finalmente solo dos docentes que es $2,27 \%$ y cinco administrativos que es el $6,67 \%$ mencionaron que dicha actitud es bajo. A manera de síntesis la actitud al cambio imperante en la universidad es el alto, o sea la mayoría del personal que labora, proponen el cambio como actitud de mejora e innovación. Ahora veamos la prueba de hipótesis.

Respecto a la prueba de hipótesis, como el diseño utilizado fue el descriptivo - correlacional, el estadígrafo utilizado fue el coeficiente de correlación de Spearman, prueba bilateral o a dos colas. Ahora bien, como la rho=0,946 y ( $p$-valor: $0,000<0,010$ ), entonces se rechaza la $H_{0}$, y se acepta la hipótesis alterna $\mathrm{H}_{1}$; concluyéndose que existe correlación directa muy fuerte y altamente significativa entre el compromiso organizacional y actitud al cambio en el personal que labora en la Universidad Nacional Intercultural de la Amazonia.

Tabla 1

Variable 1: Compromiso organizacional

\begin{tabular}{lcrrrrr}
\hline Niveles & Docentes & Porcentaje & Administrativos & Porcentaje & Total & Porcentaje \\
\hline Bajo & 1 & $1,14 \%$ & 2 & $2,67 \%$ & 3 & $1,84 \%$ \\
Regular & 66 & $75,00 \%$ & 45 & $60,00 \%$ & 111 & $68,10 \%$ \\
Alto & 21 & $23,86 \%$ & 28 & $37,33 \%$ & 49 & $30,06 \%$ \\
\hline Total & 19 & $100,00 \%$ & 75 & $100,00 \%$ & 163 & $100,00 \%$ \\
\hline
\end{tabular}

Tabla 2

Variable 2: Actitud al cambio

\begin{tabular}{lcrrrrr}
\hline Niveles & Docentes & Porcentaje & Administrativos & Porcentaje & Total & Porcentaje \\
\hline Bajo & 2 & $2,27 \%$ & 5 & $6.67 \%$ & 7 & $4.29 \%$ \\
Regular & 16 & $18,18 \%$ & 12 & $16.00 \%$ & 28 & $17.18 \%$ \\
Alto & 70 & $79,55 \%$ & 58 & $77.33 \%$ & 128 & $78.53 \%$ \\
\hline Total & 88 & $100,00 \%$ & 75 & $100,00 \%$ & 163 & $100,00 \%$ \\
\hline
\end{tabular}

Tabla 3

Coeficiente de correlación de la hipótesis general

\begin{tabular}{lllrr} 
& & & $\begin{array}{c}\text { Compromiso } \\
\text { organizacional }\end{array}$ & \multicolumn{1}{c}{$\begin{array}{c}\text { Actitud al } \\
\text { cambio }\end{array}$} \\
\hline \multirow{4}{*}{ Rho de } & Compromiso & Coeficiente de correlación & 1,000 & $0,946^{* *}$ \\
Spearman & Sig. (bilateral) & & 0,000 \\
& Actitud al & Coeficiente de correlación & $0,946^{* *}$ & 163 \\
& cambio & Sig. (bilateral) & 0,000 & 1,000 \\
& & $N$ & 163 & 163 \\
\hline
\end{tabular}

**. La correlación es significativa al nivel 0,01 (bilateral) 


\section{DISCUSIÓN}

De las tablas visualizadas de nuestra hipótesis general se deduce que el comportamiento organizacional tiene una relación directa muy fuerte y altamente significativo con la actitud al cambio; según Oseda, et al (2015) el valor del coeficiente de correlación de las variables de estudio, se rechaza la hipótesis nula y se acepta la hipótesis alterna con un ( $p$-valor: $0,000<0,010)$.

Los resultados se corroboran con lo planteado con Andrade, Betanzos, \& Paz (2006), quienes concluyen que el compromiso organizacional es un constructo básicamente actitudinal que implica sentimiento de apego y de mantener un comportamiento consistente para evitar perder los beneficios de la organización. Además, proponen una dimensión más de identificación - implicación, la que amplía la comprensión de la variable.

También se tiene en cuenta los aportes de Uribe (2007) cuando concluye que las variables de estudio se relacionan y además no se encuentra diferencias considerables entre las organizaciones; y sugieren estrategias para mejorar las reacciones frente a los cambios en relación a los grupos encontrados.

Así mismo García, Rojas \& Díaz (2011) quienes concluyen que existen correlaciones significativas entre las distintas dimensiones de ambas escalas. Es este estudio se concluye que los empleados de la organización manifestaron una actitud de favorabilidad y flexibilidad hacia el cambio.

En la misma situación, la percepción del personal de la universidad es similar, entre docentes y administrativos a los encontrados en estas conclusiones; la mayoría de ellos perciben que el compromiso organizacional es regular y la actitud al cambio si es muy alta, pues necesitan con suma urgencia para así poder dar un nuevo rumbo a la universidad.

Al respecto, Chávez, Cigüeñas \& Martensen (2016), concluyen que el estilo de liderazgo transformacional favorece la actitud de aceptación al cambio; previniendo que el estilo de liderazgo pasivo-evitador se relaciona de manera significativa, moderada e inversa con la actitud de aceptación al cambio organizacional.

Por su lado Ávila (2014), concluye que existe relación significativa entre la actitud hacia el cambio organizacional y gestión de conflictos en las direcciones de las organizaciones. Resaltando la labor de los directivos en la actitud al cambio.

Se tiene como conclusiones que:

1. Se ha determinado con un nivel de significancia del $5 \%$ que existe una correlación directa muy fuerte ("rho" $=0,946$ ) y altamente significativo (p-valor: $0,000<0,010$ ) entre el compromiso organizacional y la actitud al cambio en los docentes de la Universidad Nacional Intercultural de la Amazonia de Pucallpa; es decir a un mejor compromiso organizacional, existe también una mejor actitud al cambio en el personal que labora en la universidad y viceversa.

2. El nivel predominante de la variable Compromiso organizacional, a nivel de docentes es que el $75 \%$ perciben que el compromiso organizacional es regular, y a nivel de administrativos el $60 \%$ menciona tambien el nivel regular o medio.

3. El nivel predominante de la variable Actitud al cambio, a nivel de docentes el 79,55\% perciben que la actitud al cambio es alta, y a nivel de administrativos el $77,33 \%$ menciona tambien que el nivel de actitud al cambio es alta y necesaria.

\section{REFERENCIAS BIBLIOGRÁFICAS}

Andrade, P., Betanzos, N., \& Paz, F. (2006). Compromiso organizacional en una muestra de trabajadores mexicanos. Psicología del Trabajo y de las Organizaciones. Volumen 22 (1), pp. 25 - 43. Recuperado de: http://www.redalyc.org/ articulo.oa? id $=231317045002$

Arenas P. y Daryelis, M. (2013). La resistencia al cambio y el aprendizaje organizacional innovador en Instituciones Educativas. (Tesis para optar el grado de Magíster). Universidad del Zulia. Recuperado de: http://tesis.luz.edu.ve/tde_arquivos/177/TDE2014-05-21T16:01:24Z-4809/Publico/arenas patino_daryelis_maria.pdf

Arias, K., Varela, I., Loli, H. \& Quintana, J. (2013) Administración de recursos humanos. México DF: Mc Graw Hill.

Ávila, I. (2014). Actitudes hacia el cambio organizacional y gestión de conflictos en directores de II.EE. públicas de San Juan de Lurigancho-Lima, año 2014. (Tesis para optar el grado de Doctora). Universidad César Vallejo. Recuperado de: http://repositorio.ucv.edu.pe/ bitstream/handle/UCV/4356/\%C3\%81vila_MIC. pdf? sequence $=1$ \&isAllowed $=y$

Chávez R., J., Cigüeñas E., M. \& Martensen M., R. (2016). La relación entre los estilos de liderazgo y las actitudes ante el cambio organizacional en una empresa de servicios. (Tesis de maestría). Universidad del Pacífico, Lima, Perú. Recuperado de http://repositorio.up.edu.pe/handle/1 1354/1586

Fernández L., M. (2016). Responsabilidad social corporativa estratégica de los recursos humanos basada en alto compromiso y resultados 
organizativos: un modelo integrador. (Tesis para optar el grado de Doctora). Universidad Complutense de Madrid. Recuperado de: http:// eprints.ucm.es/40615/1/T38184.pdf

García R., M., Rojas, M. \& Díaz, S. (2011). Relación entre el cambio organizacional y la actitud al cambio en trabajadores de una empresa de Bogotá. Diversitas: Perspectivas en Psicología, 7 (1), 125-142. Recuperado de: http://www.redalyc. org/pdf/679/67922583009.pdf

Hernández, R., Fernández, C. y Baptista, P. (2014), Metodologia de la investigación. México DF: Mc Graw Hill.
Kerlinger, F. y Lee, H. (2002). Investigación del comportamiento. México: Interamericana.

Oseda, D., Chenet, M., Hurtado, D., Chávez, A., Patiño , A., \& Oseda, M. (2015). Metodología de la investigación. Huancayo: Soluciones Gráficas S.A.

Ruíz, R. (2013). Administración del talento humano. México DF: Mc Graw Hill.

Uribe, K. (2007) Actitud frente al cambio y su relación con el compromiso organizacional en organizaciones de la región del Maule. Santiago de Chile: Universidad de Chile. 